\title{
La campagne Cops : genèse et cycle de vie de la convection en région montagneuse
}

\section{Résumé}

L'étude des précipitations convectives induites par l'orographie (Cops selon l'acronyme anglais) est un projet international coordonné qui comprend une campagne d'observations de terrain et un programme scientifique et qui vise à améliorer la qualité des prévisions des précipitations orographiques convectives à la fois par l'observation quadridimensionnelle et la modélisation de leur cycle de vie. La campagne de terrain s'est déroulée en juin-juilletaoût 2007 sur l'est de la France et le sud-ouest de l'Allemagne. Son objectif majeur était de fournir un jeu d'observations météorologiques, in situ et à distance, aussi complet que possible et sur toute la profondeur de la colonne troposphérique. A près une brève revue d'ensemble du projet, l'article se concentre sur la contribution française à la campagne $C$ ops, décrit le dispositif expérimental mis en œuvre et met en valeur quelques observations clés.

\section{Abstract}

The COPS field experiment: initiation and life cycle of convection in mountainous area

The Convective and Orographicallyinduced Precipitation Study (COPS) is a coordinated international project, comprised of an observational field campaign and a research programme aiming to advance the quality of fore casts of orographically-induced precipitation by four-dimensional observations and modelling of its life cycle. The COPS field campaign took place during J une-J uly-August 2007 over eastern France and south-western Germany. Its main objective was to provide an unprecedented comprehensive set of in situ and remotely-sensed meteorological observations of the entire depth of the troposphere.After a short overview of the project, the paper focuses on the French contribution to the COPS field phase, describes the experimental setup and highlights some key observations.

\author{
E. Richard( ${ }^{(1)}$, C. Flamant ${ }^{(2)}$, F. Bouttier( ${ }^{(3)}$, J. Van Baelen ${ }^{(4)}$, \\ C. Champollion ${ }^{(5)}$, S. Argence ${ }^{(1)}$, J. Arnault ${ }^{(1)}$, C. Barthlott $^{(6)}$, \\ A. Behrendt ${ }^{(7)}$, P. Bosser ${ }^{(8)}$, P. Brousseau ${ }^{(3)}$, J.-P. Chaboureau ${ }^{(1)}$, \\ U. Corsmeier( ${ }^{(6)}$, J. Cuesta ${ }^{(9)}$, P. Di Girolamo ${ }^{(10)}, M$. Hagen ${ }^{(1)}$, \\ C. Kottmeier(6), P. Limnaios ${ }^{(12)}$, F. Masson ${ }^{(13)}$, G. Pigeon ${ }^{(3)}$, Y. Pointin ${ }^{(4)}$, \\ F. Tridon ${ }^{(4)}$, Y. Seity ${ }^{(3)}$ et V. Wulfmeyer ${ }^{(7)}$
}

(1) Laboratoire d'aérologie (LA), CNRS et université de Toulouse 14, avenue Belin - 31400 Toulouse evelyne.richard@aero.obs-mip.fr

(2) Service d'aéronomie (SA), CNRS et université Pierre-et-Marie-Curie

(3) Centre national de recherches météorologiques (CNRM), Météo-France

(4) Laboratoire de météorologie physique (Lamp), CNRS et université Blaise-Pascal

(5) Géosciences Montpellier (GM), université de Montpellier 2

(6) Institut für Meteorologie und Klimaforschung, Karlsruhe Institute of Technology (KIT), Karlsruhe, Allemagne

(7) Institut für Physik und Meteorologie, Universität Hohenheim, Stuttgart, Allemagne

(8) Institut géographique national (IGN)

(9) Laboratoire de météorologie dynamique (LMD), CNRS et université Pierre-et-Marie-Curie

(10) DIFA, Università degli Studi della Basilicata, Potenza, Italie

(11) Deutsches Zentrum für Luft und Raumfahrt (DLR), Wessling, Allemagne

(12) Direction interrégionale du Nord-Est (DIRNE), Météo-France

(13) École et observatoire des sciences de la Terre (Eost), CNRS et université de Strasbourg

a prévision quantitative des précipitations $(P Q P)$ en régions montagneuses reste encore un grand défi posé à la communauté des sciences atmosphériques. Ces régions sont des zones à risque puisque l'accroissement des précipitations par l'orographie peut y conduire à de fortes et rapides inondations. Toutefois, les différents mécanismes qui conduisent au déclenchement des précipitations en zone de relief et surtout leur importance relative ne sont encore ni bien compris ni bien modélisés. L'analyse des prévisions opérationnelles révèle en effet de bien piètres performances quant à la prévision de l'occurrence des épisodes pluvieux et montre que ces prévisions exhibent encore des défauts systématiques tels qu'une mauvaise distribution amont-aval des quantités de pluie ou encore un déclenchement trop précoce des précipitations convectives.

Plusieurs facteurs sont généralement invoqués pour expliquer ces mauvaises performances :
- une trop grande imprécision des conditions initiales des modèles due à une mauvaise qualité ou un nombre insuffisant d'observations, cela est particulièrement vrai pour le champ d'humidité qui présente une forte variabilité spatiale ;

- une utilisation sous-optimale des observations existantes ou le manque de systèmes d'assimilation capables d'intégrer des observations existantes mais non-standard ;

- des imperfections dans les modèles eux-mêmes telles qu' une trop faible résolution spatiale ou une mauvaise représentation (on parle aussi de paramétrisation) des processus physiques.

Devant ce constat, la communauté scientifique s'est mobilisée autour d'une initiative allemande pour lancer un programme ambitieux visant à progresser significativement en matière de $P Q P$. Un élément clé de ce programme a été la réalisation de la campagne Cops (Convective and Orographicallyinduced Precipitation Study) qui s'est 
principalement intéressée aux préci pitations convectives estivales (les moins bien prévues !) et dont les objectifs visaient à :

- établir un jeu de données quadridimensionnel de l'ensemble des variables météorologiques troposphériques (aérosols inclus) ;

- développer des méthodes d'assimilation permettant de mieux initialiser les variables du cycle de l'eau dans les modèles qui résolvent la convection ;

- recueillir une documentation complète de la condition de surface et de son évolution spatio-temporelle ;

- élaborer de nouvelles paramétrisations et en évaluer l'impact sur l'amélioration de la PQP.

L a campagne Cops s'est déroulée de juin à août 2007 sur le sud-ouest de I'A llemagne et I'est de la France. La zone sélectionnée est représentée figure 1 . Elle recouvre un domaine de $250 \mathrm{~km} \times 170 \mathrm{~km}$ et comporte trois reliefs remarquables : les Vosges, la Forêt-N oire et le J ura souabe. En été, cette zone est particulièrement propice aux épisodes convectifs qui, selon la climatologie, s'y produisent presque un jour sur deux.

\section{Vue d'ensemble du dispositif expérimental}

Pour répondre aux objectifs de Cops, il était indispensable de disposer d'observations permettant d'appréhender l'intégralité du cycle de vie des systèmes précipitants, depuis les caractéristiques de I'environnement préconvectif jusqu'à la phase mature, voire l'extinction du système précipitant. Un effort important a donc été consenti pour mettre en œuvre un dispositif ambitieux permettant de fournir les observations adéquates à haute résolution spatiotemporelle et sur I'ensemble du domaine. L es mesures ont porté sur une vaste gamme de paramètres : les variables météorologiques classiques (pression, vent, température et humidité), mais également des variables ayant trait aux aérosols et aux hydrométéores, ou encore des variables permettant de caractériser les bilans énergétique et hydrique de la surface.

\section{Le dispositif instrumental déployé (voir} figure 1) a reposé sur :

- I'implantation de cinq supersites instrumentés de manière homogène, disposés
Figure 1 - Carte montrant la topographie dans le domaine Cops, ainsi que certains des éléments fixes du dispositif expérimental Cops. Les cercles noirs représentent l'extension de la couverture des radars polarimétriques (le radar opérationnel de Montancy, France, et Poldirad du DLR, Centre aérospatial allemand installé près de Strasbourg). La ligne rouge pointillée représente le transect de supersites : Vosges (V), Rhin (R), Hornisgrinde (H), Murg (M) et Stuttgart (S)

le long d'un transect perpendiculaire au sillon rhénan et permettant ainsi d'analyser les transformations des masses d'air au cours de leur déplacement d' ouest en est dans la région Cops ;

- I'extension de la couverture radar avec l'installation du radar en bande $C^{(1)}$ Poldirad du DLR (Deutsches Zentrum für Luft und Raumfahrt) près de Strasbourg, permettant notamment de restituer la nature des hydrométéores ;

- la densification de réseaux mésoéchelle opérationnels ou de recherche (stations $\mathrm{GPS}^{(2)}$, stations météorologiques et de flux en surface, et stations de mesure de I'humidité des sols) ;

- la mise en œuvre de moyens mobiles (radar Doppler en bande X sur camion, et stations de sondage atmosphérique) ; - I'échantillonnage systématique de la basse troposphère par un ensemble $d$ 'avions de recherche, les petits porteurs étant dédiés à la description de l'état de l'atmosphère à l'échelle des vallées et les plus gros porteurs, comme par exemple le Falcon 20 de Safire ${ }^{(3)}$, étant dédiés à la description de l'état de l'atmosphère à mésoéchelle et à grande échelle.

\section{Nature et objectifs de la contribution française}

La communauté française impliquée dans Cops (Cops-FR) a largement contribué au dispositif expérimental international. Elle a assuré, d'une part, I'instrumentation d'un supersite dans les Vosges ; d'autre part la mise en œuvre des opérations aéroportées effectuées à partir du Falcon 20 de Safire et la densification du réseau de stations GPS. Elle s' est par ailleurs associée à l'effort international de prévision du temps à très haute résolution spatiale.
La mise en œuvre du réseau de stations GPS et du Falcon 20 (avec à son bord le lidar embarqué L eandre 2 pour la mesure de la vapeur d'eau troposphérique, B runeau et al., 2001) visait à mieux caractériser la variabilité de la vapeur d'eau à mésoéchelle dans la région du sillon rhénan en condition préconvective et postconvective, alors que l'installation du supersite $s$ 'adressait davantage à la caractérisation du cycle de vie des systèmes précipitants dans la région des Vosges du N ord.

L es diverses expertises présentes au sein de la communauté Cops-FR ainsi que la qualité des jeux de données acquises pendant la campagne devraient permettre $d$ 'analyser, à partir d'une approche couplée observations-modélisation, quelques-uns des processus clés de l'étude du déclenchement de la convection dans la région des Vosges et de la Forêt-Noire, comme par exemple l'impact de l'humidité des sols, des hétérogénéités de surface et de l'orographie sur I'hétérogénéité des champs thermodynamiques dans la couche limite atmosphérique. Les observations

(1) Les termes bande $C, X$ ou $K$ font référence à la fréquence d'émission du radar. Plus la fréquence est élevée, plus le radar est sensible, mais en contrepartie plus l'atténuation du signal est forte et plus réduite est la portée du radar. Le radar en bande C Poldirad a une portée d'environ $100 \mathrm{~km}$ contre $20 \mathrm{~km}$ pour le radar en bande X du Lamp. (2) Global Positionning System. U n réseau de stations GPS permet de restituer une information sur la variabilité horizontale du contenu intégré en vapeur d'eau atmosphérique à l'échelle du réseau (D oerflinger, 2001). Sous certaines conditions, et au travers d'une approche tomographique (voir encadré p. 35), un tel réseau permet également de restituer une information résolue verticalement, c'est-à-dire la distribution de la densité de vapeur d'eau dans I'atmosphère (Champollion et al., 2005).

(3) Service des avions français instrumentés pour la recherche en environnement, http://www.safire.fr. 
de vapeur d'eau troposphérique seront en outre d'un intérêt majeur pour la validation des modèles à haute résolution et la mise au point de méthodes d'assimilation à l'échelle convective.

Un autre objectif, propre à la communauté Cops-FR, est de contribuer à la qualification des produits opérationnels vapeur d'eau de l'instrument spatial Iasi ${ }^{(1)}$, en s' appuyant sur les nombreuses mesures de rapport de mélange de vapeur d'eau ou de contenu intégré en vapeur d'eau acquises sur le domaine Cops dans le cadre des trois mois de la campagne.

\section{Le supersite des Vosges}

Les objectifs de la communauté Cops-FR ont conduit au déploiement d'un supersite à l'est des Vosges et à l'ouest du Rhin (figure 1). Ce site a été instrumenté de manière à respecter une certaine homogénéité avec les autres supersites plus à l'est. Chacun des supersites était équipé d'au moins une station de radiosondage, d'une station GPS, d'une station météorologique (variables

Tableau 1 - Instrumentation sur le supersite V. moyennes et turbulentes) et d'une station de mesure de l'humidité des sols. Ce dispositif était complété par un ensemble de capteurs in situ et d'instruments de télédétection actifs (radars, lidars) et passifs (radiomètres). La combinaison de cette instrumentation (à géométrie légèrement variable suivant les sites) a été définie de manière à permettre l'observation du cycle de vie des systèmes précipitants - depuis l'environnement préconvectif (à l'aide des lidars, notamment) jusqu'à I' apparition des précipitations (à l'aide des radars) sans négliger le besoin en caractérisa- tion de l'aérosol et des propriétés microphysiques des nuages et des hydrométéores.

L'instrumentation du supersite des Vosges (supersite $V$ ) a été répartie en deux sites principaux : le site de M eistratzheim (dans la vallée) où était réunie la majorité des instruments et le

(1) Infrared Atmospheric Sounding Interferometer : instrument embarqué sur la plateforme Met0 p, satellite météorologique européen en orbite polaire lancé en 2006.

\section{SUPERSITE VOSGES à Meistratzheim (48.4 ${ }^{\circ} \mathrm{N} ; 7^{\circ} 5^{\circ}$; $\left.161 \mathrm{~m}\right)$}

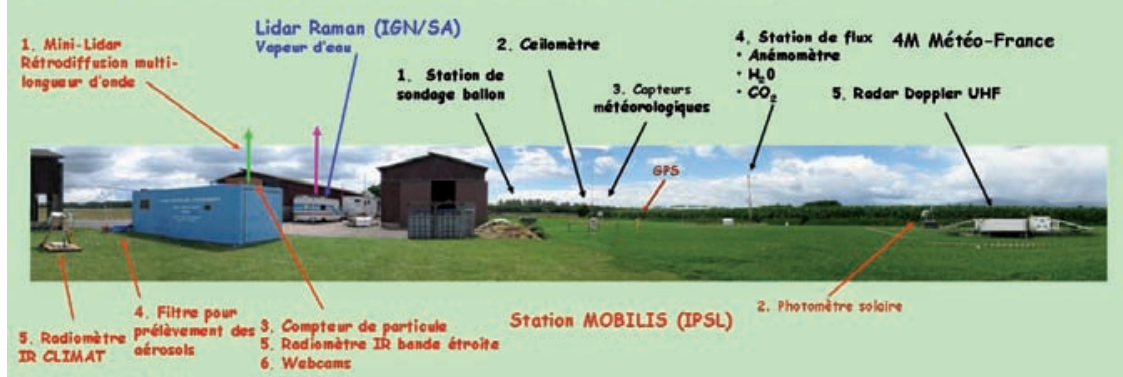

Figure 2 - Vue d'ensemble du dispositif expérimental sur le supersite V (à Meistratzheim, lieu-dit le Niedernott) dans la vallée du Rhin. Les instruments déployés par l'équipe 4M de Météo-France sont listés en noir, et ceux déployés dans le cadre de l'implémentation de la station Mobilis de l'IPSL sont listés en rouge. Les flèches verte et violette matérialisent les lignes de visée du minilidar (opérant dans le visible) et du lidar Raman (opérant dans le proche UV), respectivement.

\begin{tabular}{|c|c|c|c|c|}
\hline Instrument & Fonctionnement & Mode de visée & Période d'opération & Emplacement \\
\hline Lidar Raman de I'IGN-SA & Sur POI & Vertical ou incliné & $1^{\mathrm{er}}$ juillet - 31 juillet & Meistratzheim \\
\hline Radar bande X du Lamp & Continu & Balayage & 15 juin - 31 août & Bischenberg \\
\hline Radar bande K du Lamp & Continu & Vertical & 15 juin - 31 août & Niedernai (E-N-E Meistratzheim) \\
\hline $\begin{array}{l}\text { Lidar aérosol } \\
\text { Station Mobilis de I'IPSL }\end{array}$ & Sur POI & Vertical & $1^{\mathrm{er}}$ juillet - $1-2$ août & Meistratzheim \\
\hline $\begin{array}{l}\text { Radiomètres IR } \\
\text { Station Mobilis de I'IPSL }\end{array}$ & Sur POI & Vertical & $1^{\mathrm{er}}$ juillet - 1-2 août & Meistratzheim \\
\hline $\begin{array}{l}\text { Filtres aérosol } \\
\text { Station Mobilis de I'IPSL }\end{array}$ & Sur POI & - & $1^{\mathrm{er}}$ juillet - 2 août & Meistratzheim \\
\hline $\begin{array}{l}\text { Photomètre solaire } \\
\text { Station Mobilis de I'IPSL }\end{array}$ & Continu & Vertical & $1^{\text {er }}$ juillet - 2 août & Meistratzheim \\
\hline $\begin{array}{l}\text { Compteur particules } \\
\text { Station Mobilis de I'IPSL }\end{array}$ & Continu & - & $1^{\mathrm{er}}$ juillet - 2 août & Meistratzheim \\
\hline Profileur UHF de Météo-France & Continu & Vertical & $1^{\mathrm{er}}-31$ juillet & Meistratzheim \\
\hline Sodar de Météo-France & & & & Meistratzheim \\
\hline Station de sondage de Météo-France & Sur POI & Vertical & $1^{\mathrm{er}}-31$ juillet & Meistratzheim \\
\hline $\begin{array}{l}\text { Station d'humidité des sols } \\
\text { de Météo-France (2) }\end{array}$ & Continu & - & $1^{\mathrm{er}}-31$ juillet & $\begin{array}{l}\rightarrow \text { Meistratzheim } \\
\rightarrow \text { mi-chemin entre l'émetteur } \\
\text { et le récepteur du scintillomètre }\end{array}$ \\
\hline $\begin{array}{l}\text { Stations de flux } \\
\text { de Météo-France (2) }\end{array}$ & Continu & - & $1^{\mathrm{er}}-31$ juillet & $\begin{array}{l}\rightarrow \text { Meistratzheim } \\
\rightarrow \text { mi-chemin entre l'émetteur } \\
\text { et le récepteur du scintillomètre }\end{array}$ \\
\hline Scintillomètre de Météo-France & Continu & - & $1^{\mathrm{er}}-31$ juillet & $4 \mathrm{~km}$ au nord de Meistratzheim \\
\hline Stations GPS (2) & Continu & Incliné & $1^{\text {er }}$ juin - 31 août & $\begin{array}{l}\rightarrow \text { Bischenberg } \\
\rightarrow \text { Niedernai (E-N-E Meistratzheim) }\end{array}$ \\
\hline
\end{tabular}


site du Bischenberg (dans la montagne) où se trouvait le radar en bande $X$ du Laboratoire de météorologie physique [Lamp] et une station GPS. Les instruments présents sur le site principal de M eistratzheim (figure 2) ont été mis en œuvre dans le cadre de l'opération de la station Mobilis de I'Institut PierreSimon-L aplace [IPSL] (Cuesta et al., 2008), du camion lidar de l'Institut géographique national-Service d'aéronomie [IGN-SA ] et des moyens mobiles de $M$ étéo-France [4M ]. Le M icro Rain Radar (M RR) en bande K du Lamp, ainsi qu'un scintillomètre appartenant à $4 \mathrm{M}$ ont également été mis en œuvre à I'écart du site principal. L'ensemble des instruments déployés pendant la campagne est détaillé dans le tableau 1. Les principes de fonctionnement des instruments de télédétection mis en œuvre sur le supersite $V$ sont décrits dans Dabas et Flamant (2002, lidars, radiométrie), dans Doerflinger (2001, GPS), dans M auprivez et Sandra (2002, profileurs de vent) et dans Van Baelen et al. (2008, radars précipitation).

\section{Le réseau GPS}

Une autre contribution de Cops-FR a consisté à renforcer le réseau de stations GPS dans la région Cops, et particulièrement sur le territoire français, de manière à optimiser I'échantillonnage de la vapeur d'eau à des fins de tomographie à mésoéchelle (voir l'encadré «La restitution de la vapeur d'eau par tomographie GPS »). II s'agit d'une contribution innovante, dans la mesure où les études précédentes utilisaient la tomographie à partir d'un réseau très dense de stations GPS sur des domaines très restreints. L a faisabilité de la tomographie à mésoéchelle ainsi que l'intérêt d'une telle méthode pour l'étude de l'initiation de la convection ont récemment été mis en évidence dans le cadre du projet IHOP 2002 (International $\mathrm{H}_{2} \mathrm{O}$ Project, Champollion et al., 2009).

Figure 4 - Carte montrant l'ensemble des stations GPS participant à l'expérience Cops : les cercles rouges indiquent des stations GPS du parc Insu (https://gpscope.dt.insu. cnrs.fr), installées spécifiquement pour la campagne Cops ; les cercles blancs les stations permanentes allemandes du GFZ (German Research Centre for Geosciences) et du réseau Sapos (www.sapos.de) ; les cercles jaunes les stations permanentes du réseau SwissTopo (www.swisstopo.ch); les cercles bleus les stations des réseaux permanents RGP (Réseau géodésique français, rgp.ign.fr), Orpheon (partenaire privé) et de l'Inra Nancy (efdp.nancy-engref.inra.fr) et enfin les cercles verts les stations du réseau de l'Eost (eost.u-strasbg.fr). Les cercles pleins indiquent les stations équipées d'un système de transmission des données en temps réel. Le quadrillage blanc montre la résolution horizontale de la tomographie GPS dont on notera l'accroissement au niveau du transect est-ouest passant par les supersites.

\section{La restitution de la vapeur d'eau par tomographie GPS}

La tomographie est une méthode mathématique consistant à restituer un champ de vapeur d'eau en trois dimensions (longitude, latitude, altitude) à partir de données de vapeur d'eau en deux dimensions (c'est-à-dire intégrées sur la verticale comme le Contenu intégré en vapeur d'eau, Cive, ou Integrated Water Vapour, IWV en anglais). La tomographie GPS utilise la vapeur d'eau intégrée mesurée en oblique en direction des satellites GPS (SIWV : Slant Integrated Water Vapour). Pour chaque station GPS au sol, on peut en effet calculer la quantité de vapeur d'eau intégrée oblique en direction de tous les satellites visibles à un instant $t$ (en général une dizaine). Si l'on dispose d'un réseau suffisamment dense de GPS au sol (tous les $50 \mathrm{~km}$ ou moins), il est alors possible de restituer le champ de vapeur d'eau en trois dimensions en utilisant l'information redondante aux intersections des SIWV (figure 3). Aujourd'hui, la tomographie GPS est systématiquement réalisée en combinant les mesures GPS à des mesures
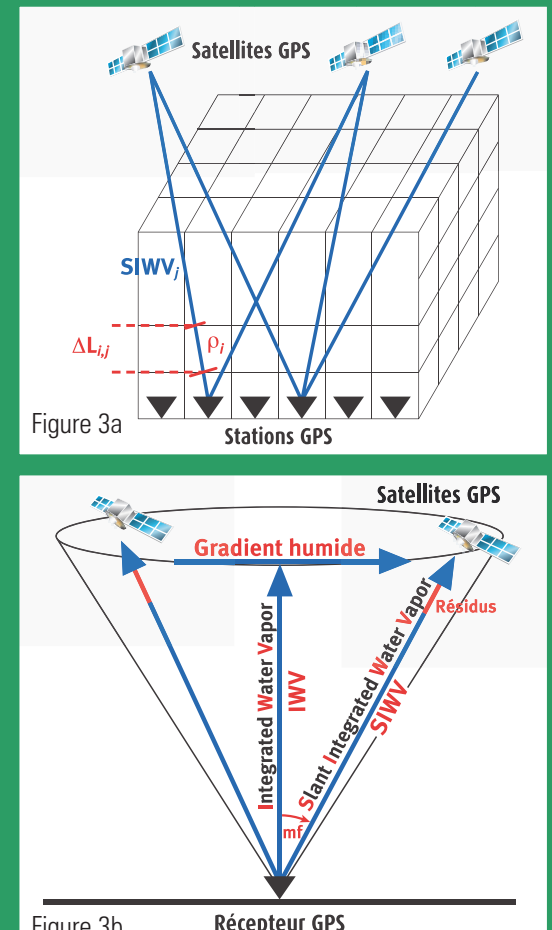

Figure $3 b$ Récepteur GPS résolues verticalement (comme les radiosondages). La tomographie permet ainsi de tirer parti des avantages de chaque instrument de mesure tout en s'affranchissant de ses limitations. Les radiosondages apportent une résolution verticale importante, mais leur nombre est limité dans le temps et dans l'espace. Le GPS apporte à l'inverse une résolution temporelle et spatiale importante, mais sans véritable résolution verticale. Au final, pendant la campagne Cops, la tomographie GPS fournit, par exemple, toutes les heures une coupe est-ouest le long des supersites avec une résolution spatiale de $10 \mathrm{~km}$ et verticale de $500 \mathrm{~m}$. Les premières comparaisons avec les mesures Lidar aéroportées montrent des résultats prometteurs.

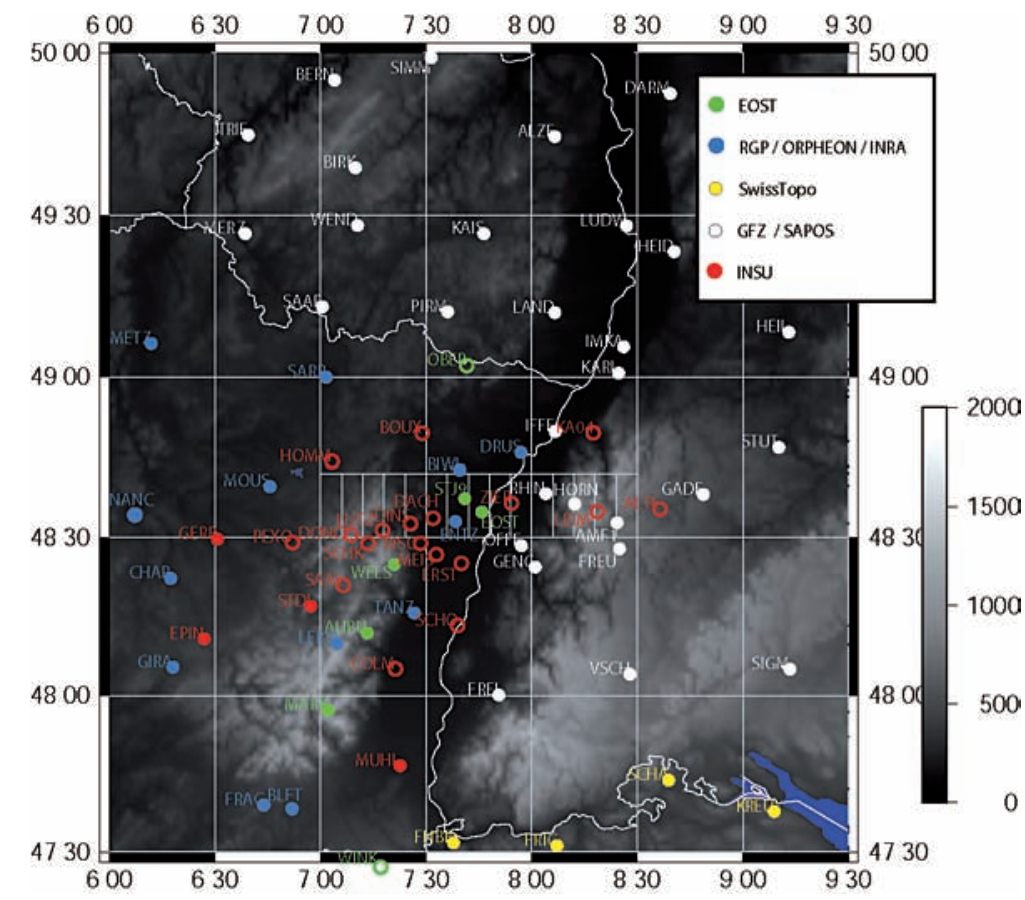


A insi, vingt-cing stations additionnelles ont été installées (vingt en France et cinq en A llemagne) et sont venues compléter les cinquante-cing stations permanentes françaises et allemandes existant dans le domaine Cops. Ce réseau densifié (figure 4) permettra de restituer des profils de rapport de mélange de vapeur d'eau avec une résolution horizontale d'environ $50 \mathrm{~km}$ et une résolution verticale de l'ordre de 500 m en continu (résolution de $60 \mathrm{~min}$.) sur les trois mois de la campagne. La densité des stations GPS a par ailleurs été davantage accrue le long de l' axe est-ouest passant par les supersites de manière à pouvoir restituer sur ce transect des profils tous les $10 \mathrm{~km}$ environ. De manière plus classique le réseau ainsi redéfini a également permis un suivi en temps réel des fluctuations de contenu intégré de vapeur d'eau à travers le domaine Cops pendant les trois mois de la campagne.

\section{Les opérations aéroportées}

La mise en œuvre du lidar à absorption différentielle L eandre 2 à bord du Falcon 20 avait pour objectif premier la mesure à haute résolution spatiale de la vapeur d' eau dans la basse troposphère, afin d'analyser les relations existant entre la variabilité du champ de vapeur d'eau à mésoéchelle et l'initiation de la convection, à l'instar des études menées autour des «drylines » dans les Grandes Plaines des États-Unis dans le cadre du projet IHOP 2002 (Flamant et al., 2003). La stratégie expérimentale associée a consisté à échantillonner systématiquement le champ de vapeur d'eau dans la région du sillon rhénan en période préconvective, le long d'axes prédéfinis (figure 5).

Un deuxième objectif dévolu à l'instrument L eandre 2 était de constituer une base de données de référence pour l'intercomparaison des mesures de vapeur d'eau (résolues verticalement), effectuées par les instruments de télédétection (indépendamment de la technique utilisée) au cours de la campagne. Avant de combiner I'ensemble des observations de vapeur d'eau obtenues par différentes techniques (télédétection active et passive, mesure in situ), un tel exercice était indispensable.

Figure 5 - Plan de vol typique du Falcon 20 au cours de missions Cops pendant lesquelles les différents axes est-ouest puis nord-sud (lignes vertes) sont décrits séquentiellement. Les losanges rouges représentent la position des cinq supersites ainsi que la position du radar Poldirad. Les astérisques indiquent les positions de largage de dropsondes. Le triangle violet indique la position de la station de sondage de Burnhaupt (France).
La stratégie expérimentale associée a consisté à effectuer des vols coordonnés avec le Falcon 20 du DLR (égal ement équipé d' un système lidar vapeur d' eau à absorption différentielle) et survoler systématiquement les sites équipés d' instruments mesurant la vapeur d'eau ( $c^{\prime}$ est-à-dire les supersites V, R, H et M ; cf. figure 1).

Onze vols dédiés à la documentation du champ de vapeur d'eau en période préconvective et trois vols dédiés à l'intercomparaison ont été réalisés avec le Falcon 20 du Safire, entre le 9 juillet et le 2 août 2007. Outre la restitution de profils verticaux de rapport de mélange de vapeur d'eau (avec des résolutions verticale et horizontale de I' ordre de $300 \mathrm{~m}$ et $4 \mathrm{~km}$, respectivement), L eandre 2 a également permis de cartographier avec précision les couches diffusantes dans l'atmosphère (aérosols et nuages) via la mesure des profils de réflectivité atmosphérique (à $730 \mathrm{~nm}$ ) à haute résolution (15 m sur la verticale, 200 m sur I'horizontale).

\section{Modélisation et prévision}

Grâce à la coordination avec le projet MAP D-Phase (voir l'encadré «MAP $D$-Phase »), l'expérience Cops a bénéf icié d'un soutien sans précédent en matière de prévision et de modélisation. Pour la conduite de l'expérience, les scientifiques ont disposé d'un ensemble d'une dizaine de prévisions déterministes à l'échelle kilométrique et d'une hiérarchie de différentes prévisions probabilistes allant de l'échelle globale à I'échelle régionale. La communauté française s'est associée à l'effort international en mettant en œuvre deux chaî-

\section{MAP D-Phase}

Le projet MAP D-Phase (Demonstration

of Probalistic Hydrological and Atmospheric Simulation of Flood Events in the Alpine Region ; Rotach et al, 2008) est un " Forecast Demonstration Project » du World Weather Research Programme de l'Organisation météorologique mondiale. II intervient dans le prolongement de l'expérience MAP (Mesoscale Alpine Programme) dédiée à l'étude des précipitations intenses dans la zone alpine (Bougeault et al., 2001). Lors de MAP (1999), différents modèles météorologiques, de recherche pour la plupart, non-hydrostatiques et de résolution kilométrique, ont été mis au point, testés et évalués. Avec le temps, ces nouvelles techniques de modélisation ont essaimé vers les services météorologiques. MAP D-Phase a pour but d'évaluer en vraie grandeur ces nouveaux outils dans une utilisation quasi opérationnelle. Pour optimiser la valorisation des résultats, la zone et la période d'étude ont été étendues pour couvrir à la fois les intérêts de MAP (Italie du Nord, précipitations intenses automnales) et de Cops (Vosges-ForêtNoire, précipitations convectives estivales). Plus de trente modèles atmosphériques (couplés à sept modèles hydrologiques) ont participé à MAP DPhase et mis en ligne leurs résultats de juin à novembre 2007 .

nes de prévisions à très haute résolution spatiale, l'une fondée sur une version préliminaire du modèle A rome (le futur modèle opérationnel de M étéo-France ; B outtier, 2007), I' autre exploitant le modèle de recherche M éso- $\mathrm{NH}$ développé conjointement par le Centre national de la recherche scientifique (CNRS) et M étéo-France (L afore et al,

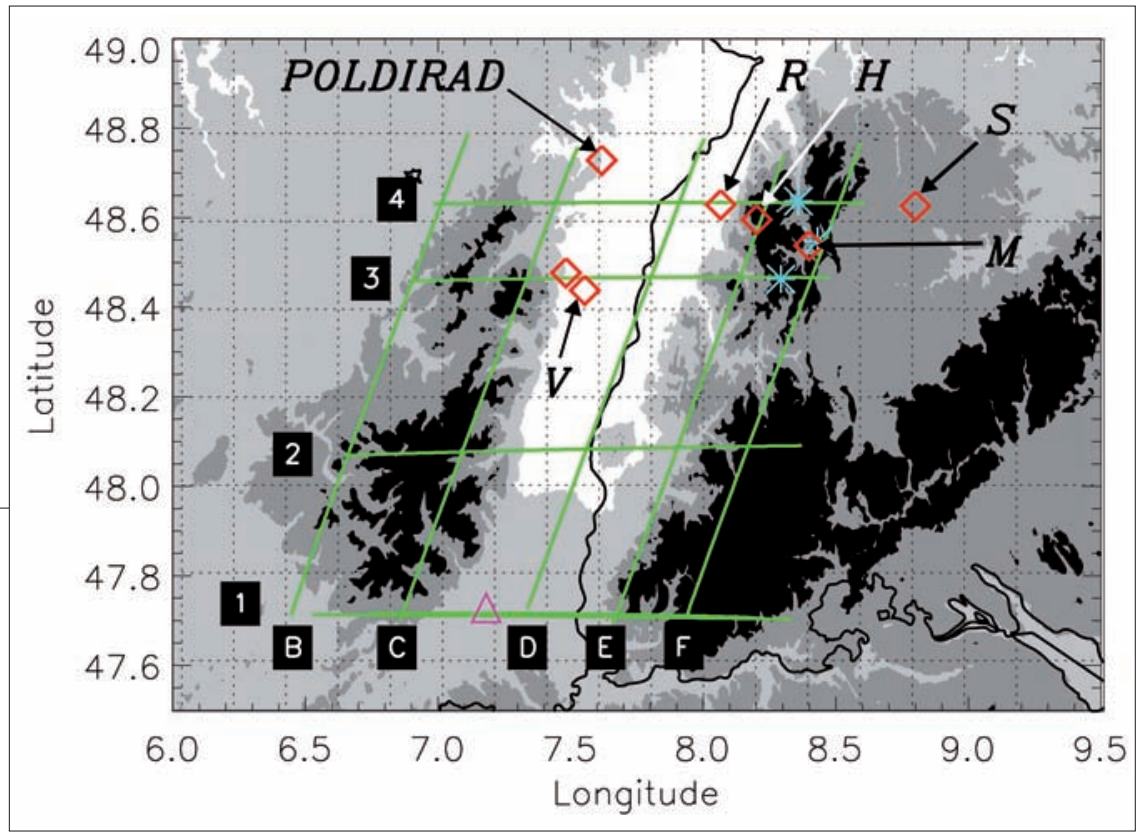


1998). Ces deux modèles partagent globalement le même jeu de paramétrisations physiques, mais différent dans leur système d'équations et leurs schémas numériques ; dans le contexte de Cops, ils ont par ailleurs utilisé des modes d'initialisation et de couplage différents. L es conditions initiales (provenant d' une analyse à $00 \mathrm{~h} 00 \mathrm{TU}$ ) et les conditions aux limites latérales (obtenues à partir de prévisions d'un modèle de plus grande échelle) ont été fournies par le modèle du Centre européen pour les prévisions météorologiques à moyen terme (CEPM M T) dans le cas de M éso$\mathrm{NH}$ et par le modèle A ladin dans le cas d'A rome. Ces deux chaînes parallèles devraient nous aider à terme à mieux discriminer les erreurs de prévisions émanant du modèle de celles induites par les conditions initiales.

\section{Les premiers résultats}

\section{Un prérequis : la validation croisée des mesures de vapeur d'eau}

L es premières comparaisons systématiques entre $L$ eandre 2 et les dropsondes (figures $6 a$ et $6 b$ ), d'une part, et entre L eandre 2 et le lidar sol Raman Basil de I'université de la Basilicata (supersite $R$, figures $6 c$ et $6 d$ ), d'autre part, ont révélé les très bonnes performances de L eandre 2 au cours de la campagne. Les différences observées entre les mesures L eandre 2 et dropsondes sont essentiellement liées à la géométrie d'observation associée à chacune des techniques. Le profil Leandre 2 est issu d'un sondage vertical, quasi instantané de l'atmosphère dans la région de largage de la droposonde, alors que le profil issu de celle-ci est obtenu en une quinzaine de minutes le long d'une trajectoire oblique fortement influencée par les vents de basses couches. A contrario, I'accord entre les profils obtenus de nuit (figure $6 \mathrm{~d}$ ) par lidar Raman et à absorption différentielle est excellent (biais quasi nul entre 1000 et 4500 m

Figure 6 - Comparaison entre profils de rapport de mélange de vapeur d'eau issus de mesures dropsondes et du système aéroporté Leandre 2, le $1^{\text {er }}$ août à 13 h 47 TU (a) et 16 h 20 TU (b). Comparaison entre profils de rapport de mélange de vapeur d'eau issus de mesures du système aéroporté Leandre 2 (rouge), du système sol Basil opéré sur le supersite $R$ (bleu) et de radiosondage (vert) pour (c) le 16 juillet (mesures de jour) et (d) le 31 juillet (mesures de nuit). au-dessus du sol). L'accord entre mesures lidar sol et lidar aéroporté est moins bon concernant les comparaisons effectuées de jour (figure $6 \mathrm{c}$ ) avec un biais compris entre -1 et $1 \mathrm{~g}_{\mathrm{kg}} \mathrm{kg}^{-1}$. Les différences peuvent être en partie imputées à une moins bonne performance des systèmes de type Raman en période diurne, et une dégradation du rapport signal sur bruit en fonction de l'altitude (exemple au-dessus de $3000 \mathrm{~m}$ ).

Ces résultats sont importants : à l'avenir, les mesures des lidars aéroportés (de loin les plus riches en termes de couverture spatiale) pourront être exploitées avec confiance et L eandre 2 pourra servir de référence pour l'exercice d'intercomparaison impliquant I'ensemble des mesures lidar vapeur d'eau. L es résultats préliminaires de l'intercomparaison entre L eandre 2 et B asil effectuée sur un
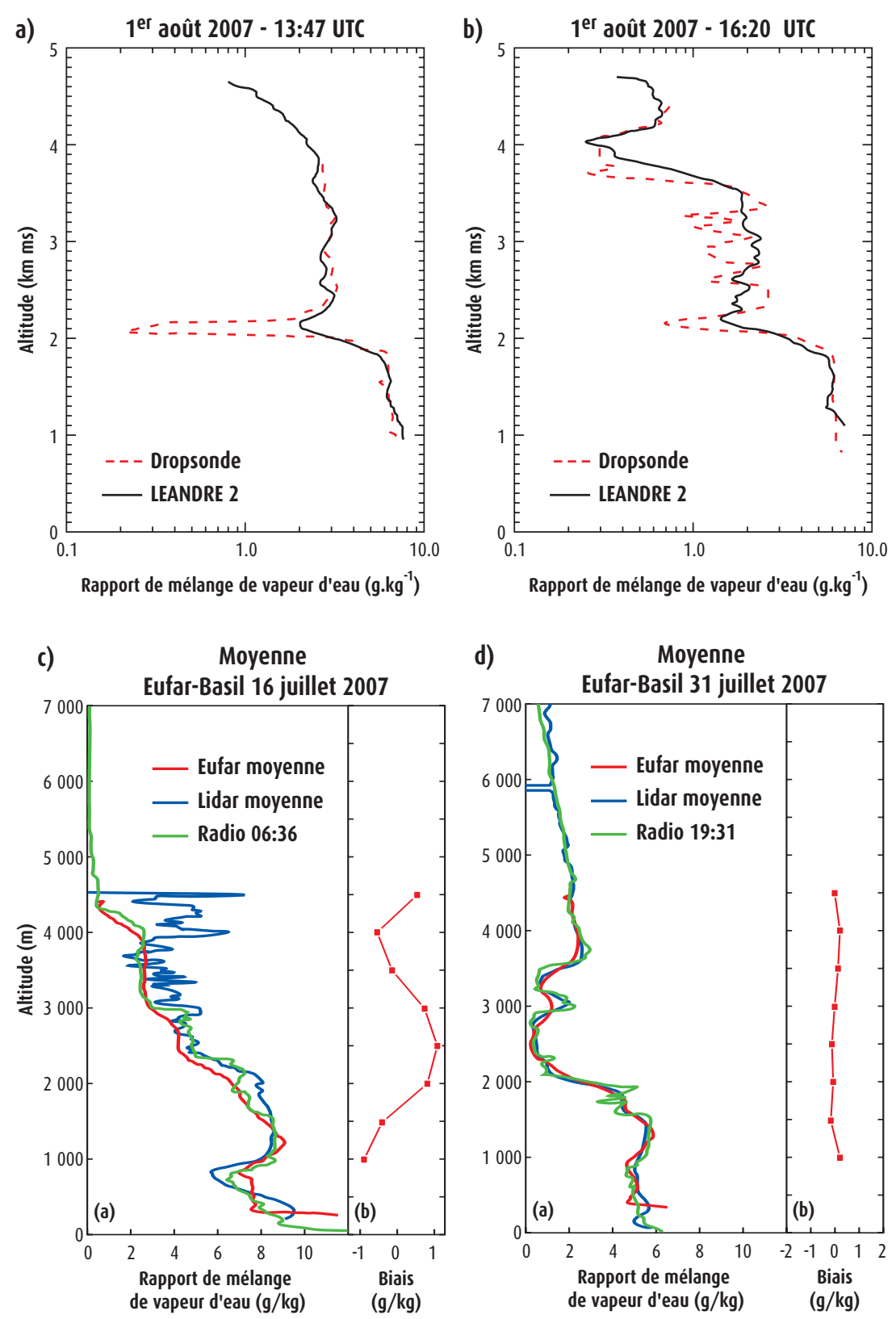

échantillon de vingt-cing coïncidences mettent en évidence un biais moyen de l'ordre de $4 \%\left(0,08 \mathrm{~g} \cdot \mathrm{kg}^{-1}\right)$ et une erreur quadratique moyenne de l'ordre de $14 \%$ $\left(1{\mathrm{~g} . \mathrm{kg}^{-1}}^{-1}\right.$ ) entre 0 et $4,5 \mathrm{~km}$.

\section{La structure complexe des champs d'humidité}

Le $1^{\text {er }}$ août, l'advection de masses d'air d'origine saharienne à l'avant d'une perturbation de moyenne latitude a contribué à augmenter la variabilité de la vapeur d'eau avant le déclenchement de la convection sur les Vosges et la Forêt-N oire suite à l'arrivée d'un front chaud sur la région Cops. Les figures 7 et 8 illustrent la complémentarité entre les mesures lidar de vapeur et de rétrodiffusion atmosphérique acquises sur le supersite $V$ et celles effectuées à bord 
Figure 7 -

a) Réflectivité atmosphérique à $532 \mathrm{~nm}$ observée par le lidar rétrodiffusion de la station Mobilis entre $18 \mathrm{~h} 00$ TU le 31 juille et $03 \mathrm{~h} 00$ TU le 2 août 2007 sur le supersite $V$.

b) Rapport de mélange de vapeur d'eau atmosphérique observé

par le lidar Raman entre 18 h 00 TU le 31 juillet et 03 h 00 TU le $1^{\text {er }}$ août.

du Falcon 20 au cours de la journée du $1^{\text {er }}$ août et de la nuit précédente.

La figure 7a montre la réflectivité atmosphérique (à $532 \mathrm{~nm}$ ) issue du lidar rétrodiffusion de la station M obilis entre $18 \mathrm{~h} 00$ TU le 31 juillet et 03 h 00 TU le 2 août 2007. Les données du lidar ont ainsi permis de documenter :

- les processus dynamiques de couche limite en phase préconvective (jusqu'à environ 15 h 00 TU le $1^{\text {er }}$ août) ;

- puis l'arrivée des masses d'air d'origine saharienne, mise en évidence notamment par I' augmentation de la réflectivité dans la troposphère libre (entre 2 et $5 \mathrm{~km}$ d'altitude) associées à la présence des aérosols désertiques (entre $15 \mathrm{~h} 00 \mathrm{TU}$ le $1^{\text {er }}$ août et 01 h 00 TU le 2 août) ;

- et enfin, I'arrivée du front chaud, identifiable par les fortes valeurs de réflectivité associées aux nuages de type cirriforme, entre 7 et $8 \mathrm{~km}$, vers 00 h 30 TU le 2 août dans un premier temps, puis par la baisse systématique de la base des nuages présents le long du front (de type altocumulus, puis al tostratus et enfin nimbostratus) jusqu'à I'apparition de la pluie sur le site vers $02 \mathrm{~h} 00 \mathrm{TU}$ le 2 août.

Les données du lidar Raman (figure 7b) montrent la complexité de la structure verticale du champ de vapeur d'eau au cours de la nuit du 31 juillet au $1^{\text {er }}$ août, et notamment la présence d'une couche très sèche (entre 2 et $3 \mathrm{~km}$ ) et caractérisée par un rapport de mélange de I'ordre de 1 à

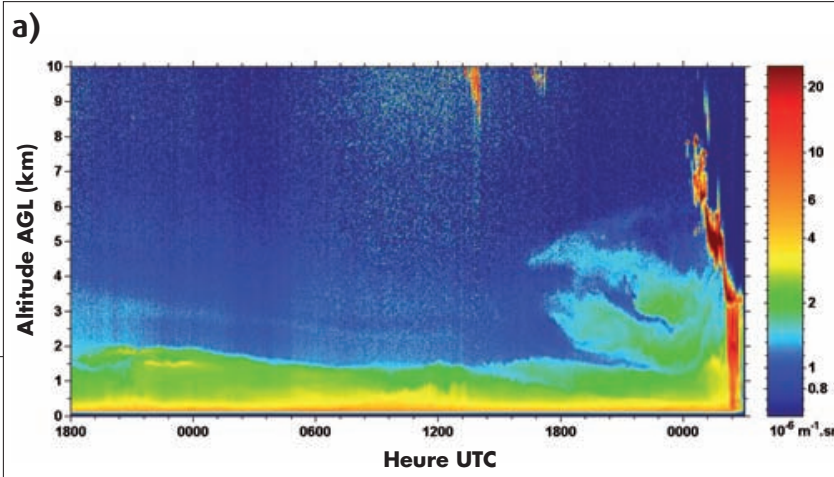

b) Lidar IGN-SA : 31 juillet 2007

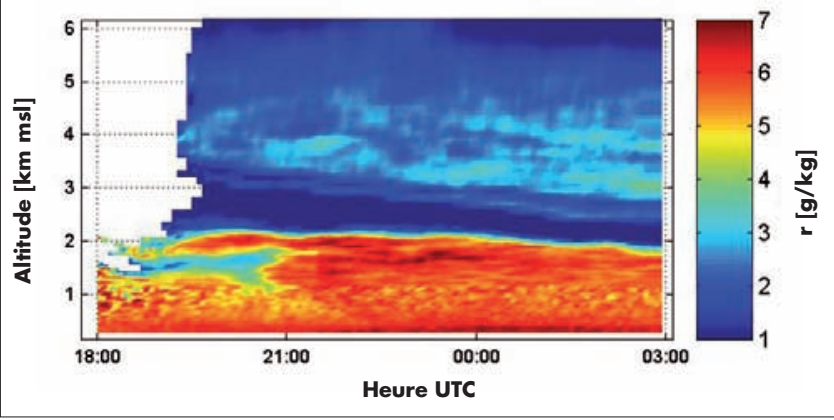

Figure 8 - a) Observations Leandre 2 le long de l'axe est-ouest numéroté 2 sur la figure 5 entre 08 h 41 et 0855 TU le $1^{\text {er }}$ août 2007.

b) Même chose que pour (a), mais entre 14 h 20 et 14 h 35 TU. En (a) et (b), sont montrées les coupes de rapport de mélange de vapeur d'eau (haut, en $\mathrm{g} / \mathrm{kg}$ ) et de réflectivité atmosphérique à $730 \mathrm{~nm}$ (bas, en unité arbitraire). L'orographie apparaît en noir.

a)

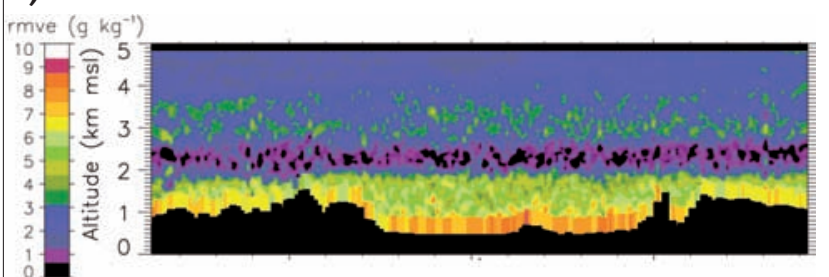

Refl (a.u.)

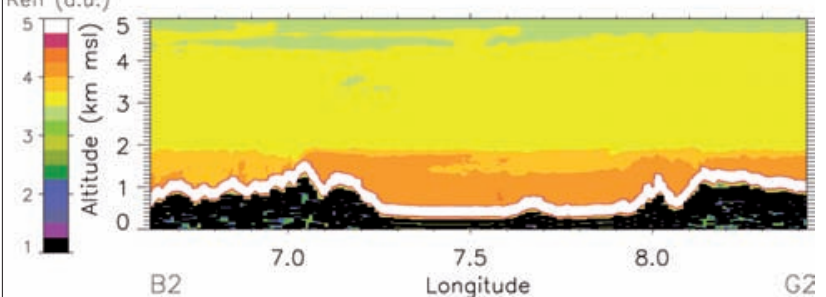

b)

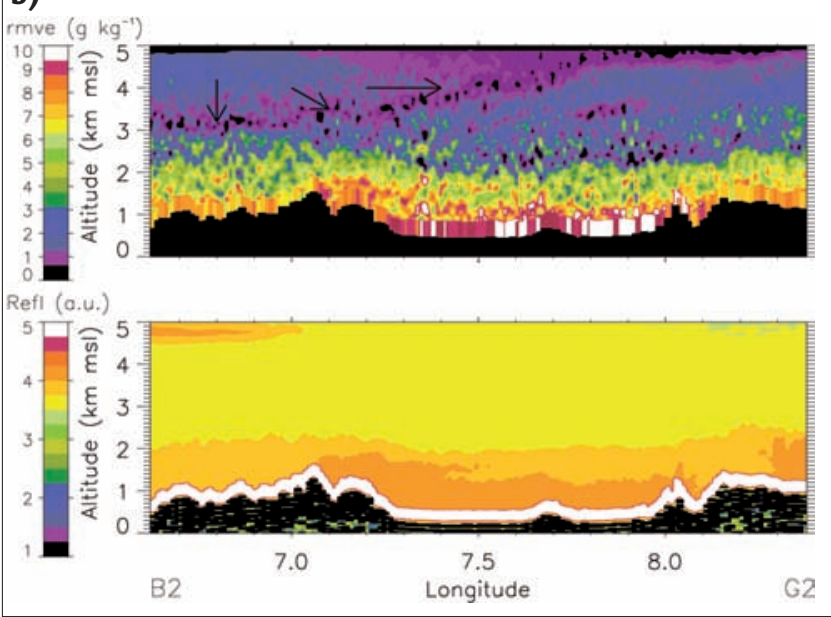

2 g.kg-1, surmontant une couche plus humide ( 5 à $\left.7 \mathrm{~g} \cdot \mathrm{kg}^{-1}\right)$, correspondant à une couche résiduelle, c' est-à-dire les reliquats de la couche limite convective s'étant développée la veille. Au-dessus de la couche sèche, on note la présence d'une couche légèrement plus humide (3 à $\left.4 \mathrm{~g} \mathrm{~kg}^{-1}\right)$.

La figure 8 a montre les observations du champ de vapeur d'eau à mésoéchelle, acquises par le lidar L eandre 2 à bord du Falcon 20 entre 08 h 40 et 08 h 55 TU le $1^{\text {er }}$ août, le long d'un axe orienté est-ouest et au sud du supersite V. Ces données mettent en évidence les trois mêmes couches que celles observées avec le lidar Raman la nuit précédente, ainsi que leur extension horizontale au travers du sillon rhénan et au-dessus du relief. Les observations, effectuées par Leandre 2 en début d'après-midi (14 h 20 $14 \mathrm{~h} 35 \mathrm{TU}$, figure 8b) sur le même axe, montrent une situation assez différente avec la quasi-disparition de la couche sèche au sommet de la couche limite, et I'apparition d'une couche très sèche à l'interface entre la masse d'air d'origine saharienne (à l' ouest du domaine, indiquée par les trois flèches sur la figure 8b) et la masse d'air à l' avant de celle-ci. Les prémices du panache de poussières désertiques observées en fin d'après midi le $1^{\text {er }}$ août au-dessus de la vallée du Rhin sont égal ement visibles sur les données de réflectivité Leandre 2 à $\mathrm{I}^{\prime}$ ouest de $7^{\circ} \mathrm{E}$ et vers $5 \mathrm{~km}$ d'altitude.

L'ensemble des données recueillies par les différentes plates-formes au cours de cette période devrait notamment permettre de mieux comprendre le rôle des advections humides de secteur sud-ouest à l'avant des perturbations de moyenne latitude sur le déclenchement de la convection dans la région des Vosges et de la Forêt-Noire. 

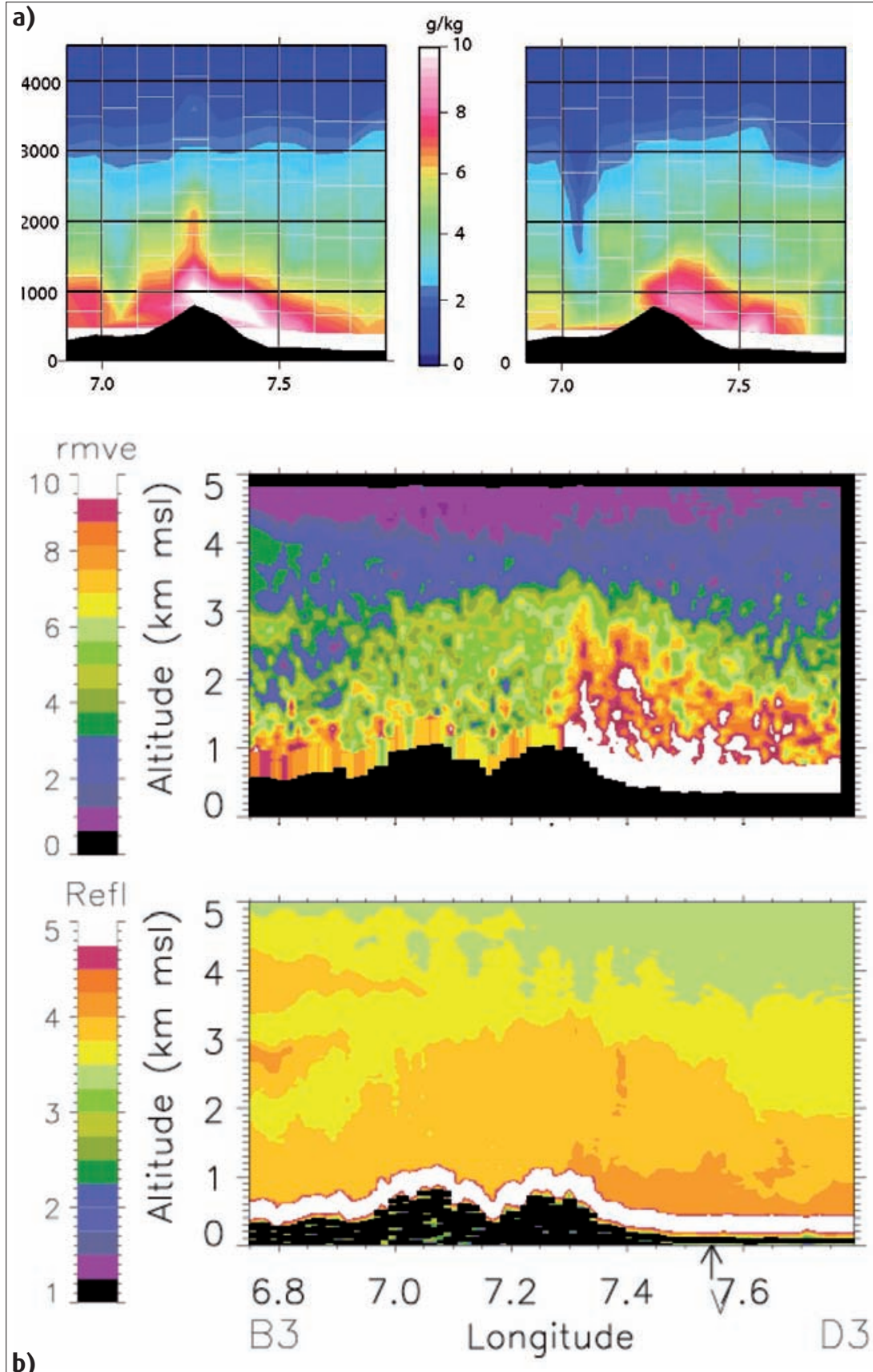

b)

\section{Les mesures \\ d'humidité par GPS}

La figure 9a présente une coupe verticale (longitude-altitude) de densité de vapeur d'eau le long d'un axe est-ouest extraite de l'analyse tomographique de la journée du 15 juillet 2007 à deux échéances au cours de l'après-midi, période durant laquelle une cellule orageuse isolée s'est développée audessus de la Forêt-Noire (cf. paragraphe suivant). Les observations GPS mettent en évidence une accumulation d'humidité sur le flan est des Vosges et au-dessus de la vallée du Rhin. Elles mettent également en exergue Ia grande variabilité temporelle du champ de vapeur d'eau dans cette région. La distribution verticale de la vapeur d'eau au-dessus des Vosges suggère l'existence d'une circulation anabatique associée au chauffage le long des pentes de la montagne. Ces observations sont corroborées par les mesures de rapport de mélange de vapeur d'eau acquises avec L eandre sur le même axe, et qui montrent aussi une accumulation d'humidité à l'est des Vosges (figure 9b). Ce comportement a été observé le long de la vallée du Rhin sur une centaine de kilomètres. L es raisons de cette accumulation de vapeur d'eau sont encore mal connues : est-elle liée à une intensification des échanges surfaceatmosphère dans la vallée ? Ou bien résulte-t-elle d'une dissymétrie est-
Figure 9

a) Densité de vapeur d'eau (coupe longitude-altitude) issue de l'analyse tomographique le long de l'axe est-ouest B3-D3 (voir figure 5 pour la localisation) le 15 juillet entre $12 \mathrm{~h} 00$ et $12 \mathrm{~h} 30$ TU (gauche) et entre 13 h 00 et 13 h 30 TU (droite).

b) Idem à la figure $8 \mathrm{a}$, mais le 15 juillet le long de l'axe B3-D3, entre 13 h 16 et 13 h 26 TU.

ouest des courants anabatiques qui se développent sur les flancs du relief sous I'influence du réchauffement diurne ? L'utilisation conjointe de la tomographie et des jeux de données plus résolus, mais aussi plus ponctuels, permettront de mieux comprendre l' influence du système convectif sur son environnement, et plus particulièrement sur la variabilité du champ de vapeur d'eau dans la région Cops.

\section{La prévision}

\section{d'un orage isolé : une question de chance?}

Les orages isolés sont particulièrement difficiles à prévoir et mettent très souvent en échec les modèles opérationnels actuels. Trois conditions sont nécessaires à leur déclenchement : une humidité suffisante dans les basses couches de l'atmosphère, une instabilité potentielle de la masse d'air et un mouvement vertical ascendant résultant, soit du réchauffement diurne du sol, soit du soulèvement orographique. Si les mécanismes déclencheurs sont connus, il est rare que leur représentation dans les modèles soit suffisamment précise pour permettre de répondre avec exactitude aux questions suivantes: où, quand, combien et pendant combien de temps va-t-il pleuvoir? Les résultats obtenus sur le cas du 15 juillet montrent cependant des résultats encourageants pour les modèles de haute résolution. $\mathrm{M}$ éso- $\mathrm{NH}$ de résolution horizontale $2 \mathrm{~km}$ (de même qu'A rome de résolution 2,5 km) a été capable de prévoir l'orage fugace, mais violent, qui s'est formé en fin $d^{\prime}$ après midi à proximité de Freudenstadt en Forêt-N oire (figure 10). Ce sont les deux seuls modèles de l'ensemble D-Phase qui sont parvenus à ce résultat. II est dès lors essentiel d'identifier et d'analyser les causes du succès ou de l'échec de ces différentes prévisions. Sont-elles imputables à la nature chaotique de l'atmosphère, auquel cas les bonnes prévisions I'ont été par chance ou bien existe-t-il un facteur déterministe qui a conditionné le succès des bonnes prévisions? 


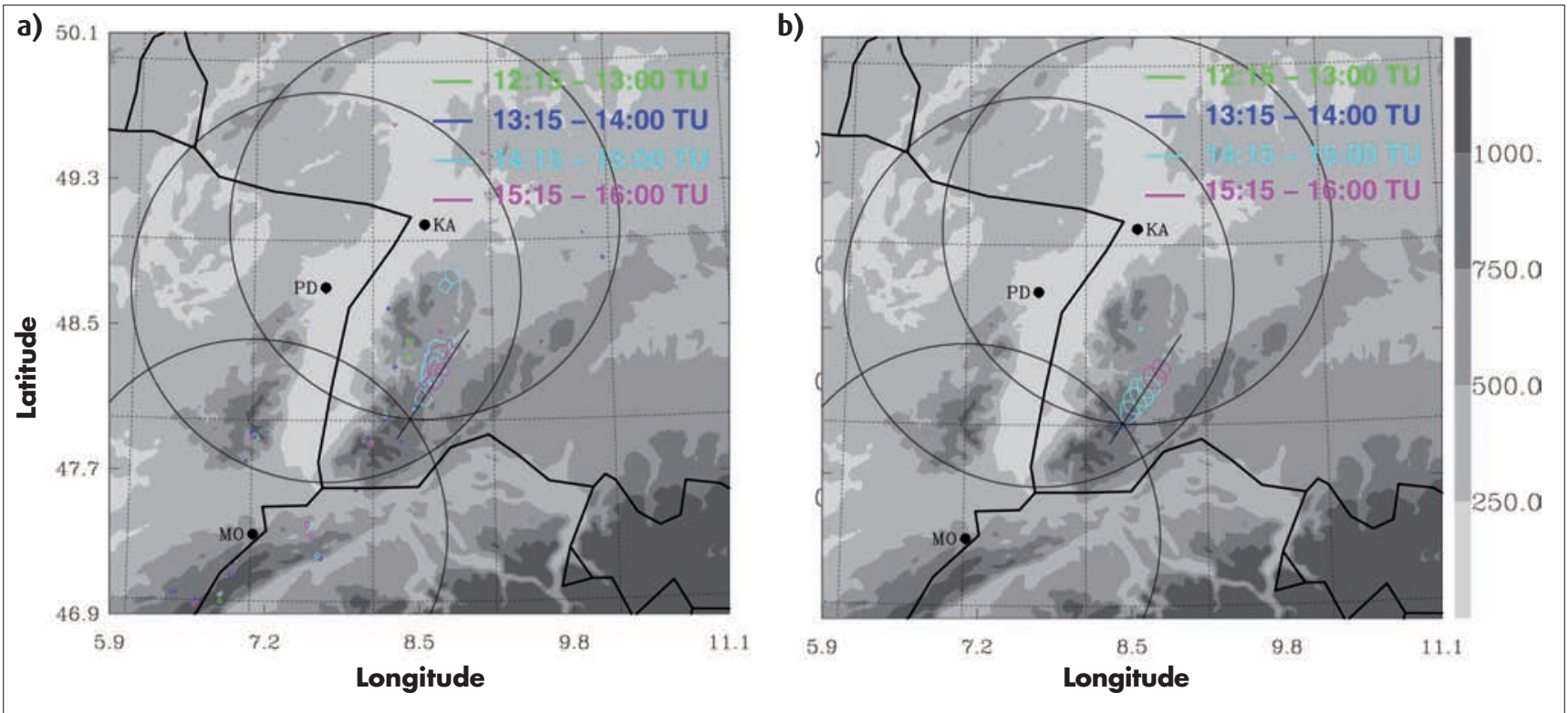

Figure 10 - Évolution temporelle de l'isocontour $1 \mathrm{~mm} / \mathrm{h}$ du champ de précipitation entre 12 h 15 TU et 16 h 00 TU : a) observée par le radar de Montancy (position indiquée par les lettres MO) ; b) simulée par Méso-NH.

Figure 11 - Trajectoire des cellules orageuses (en haut) et localisation de leur première occurrence (en bas) pour les 12 et 13 août 2007 Le cercle blanc matérialise la portée du radar en bande $\mathrm{X}$ installé sur le site du Bischenberg.

\section{La barrière du Rhin}

D'un jour à l'autre, la convection peut prendre des formes très différentes. La figure 11 en présente une illustration intéressante. Elle montre à partir des observations du radar Poldirad, pour deux journées consécutives (les 12 et 13 août 2007), la trajectoire des cellules orageuses ainsi que la position géographique de leur première occurrence. II apparaît nettement que le 12 août, la convection est initiée sur les crêtes des reliefs, alors que le 13, les développements convectifs se produisent à l'est (et sous le vent) des reliefs. On peut aussi constater que la trajectoire des cellules est beaucoup plus courte le premier jour que le second. Durant la journée du 13 , les orages vosgiens s'exportent outre-Rhin, alors que la veille, ils s'éteignent après quelques dizaines de kilomètres. Ces observations laissent penser que les mécanismes d'initiation pour ces deux journées sont assez différents. Le premier jour, il s' agit vraisemblablement d'un pilotage thermique. Le réchauffement diurne provoque des courants ascendants sur les flancs des reliefs et une convergence à la crête. L ors de la seconde journée, I' origine de I'ascendance est de nature dynamique,
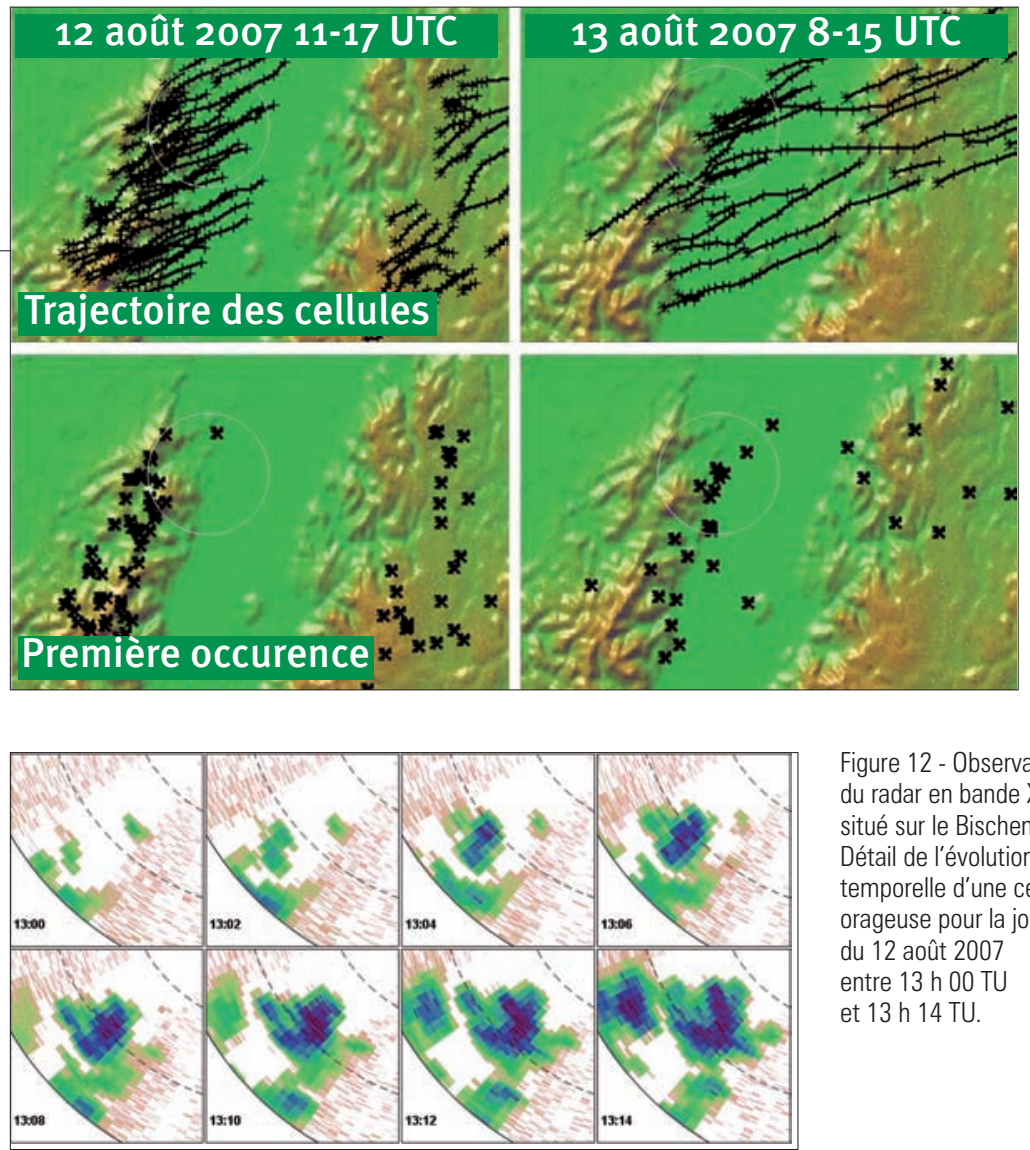

Figure 12 - Observations du radar en bande $X$ situé sur le Bischenberg. Détail de l'évolution temporelle d'une cellule orageuse pour la journée du 12 août 2007 entre $13 \mathrm{~h} 00 \mathrm{TU}$ et $13 \mathrm{~h} 14 \mathrm{TU}$.

I'écoulement de sud-ouest contourne les reliefs et forme une zone de convergence dans leur sillage.

Le radar en bande $X$, mis en œuvre par le Lamp sur le site du Bischenberg, a permis d'observer en détail I'évolution temporelle de ces cellules orageuses avec une résolution spatiale de 60 mètres et une résolution temporelle de 30 secondes. La figure 12 illustre la phase d'initiation d'une cellule qui naît dans la zone d'observation du radar aux environs de 13 h 00 TU le 12 août. Cette cellule se forme sur le flan est du "Champ du Feu », le dernier point 


\author{
Figure 13 - Évolution temporelle \\ pour juillet et août 2007 \\ de la moyenne spatiale des précipitations \\ journalières observées et prévues. \\ Les précipitations prévues \\ ont été interpolées \\ aux points de mesure et le domaine spatial \\ considéré correspond à celui \\ de la figure 9 .
}

culminant du massif vosgien juste à l'ouest du radar et avant la plaine du Rhin. Jusqu'à 13 h 08 TU, la cellule grossit sur place, alimentée par les processus convectifs. Plus tard, elle est entraînée par le vent moyen de sudouest, alors qu' une seconde cellule est générée légèrement plus en amont.

La combinaison de ces jeux de données très complémentaires est d'un intérêt crucial pour la validation des modèles numériques. Elle permet à la fois d'étudier très en détail la phase d'initiation de la convection, d'en suivre le déplacement spatial et d'appréhender son cycle de vie.

\section{L'évaluation des modèles}

L'évaluation des modèles de haute résolution et capables de résoudre explicitement la convection fait partie des enjeux majeurs de Cops. Cette évaluation sera conduite in extenso dans le cadre du projet MAP D-Phase. La figure 13 permet d'obtenir un aperçu de résultats. Elle montre I'évolution
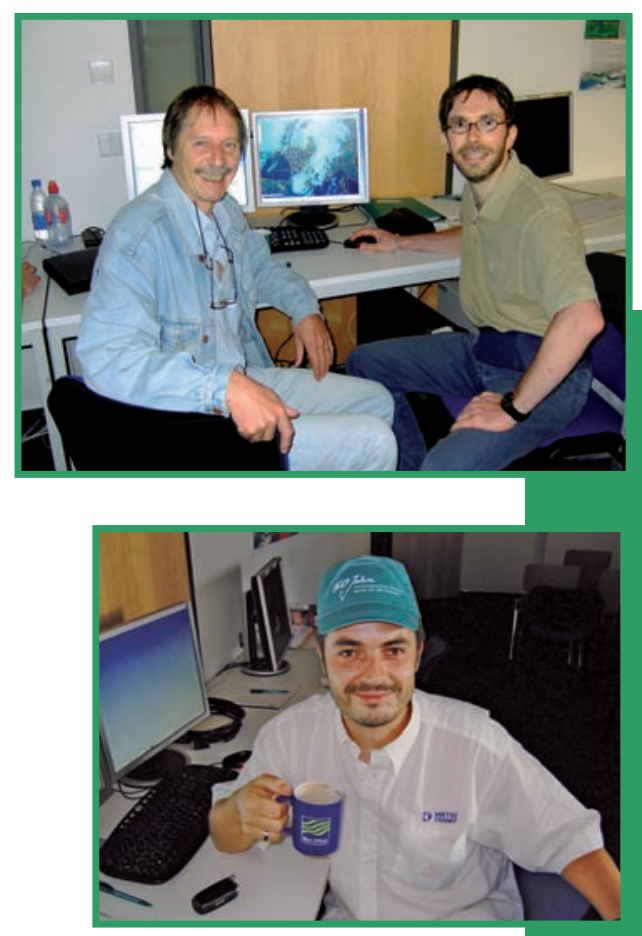
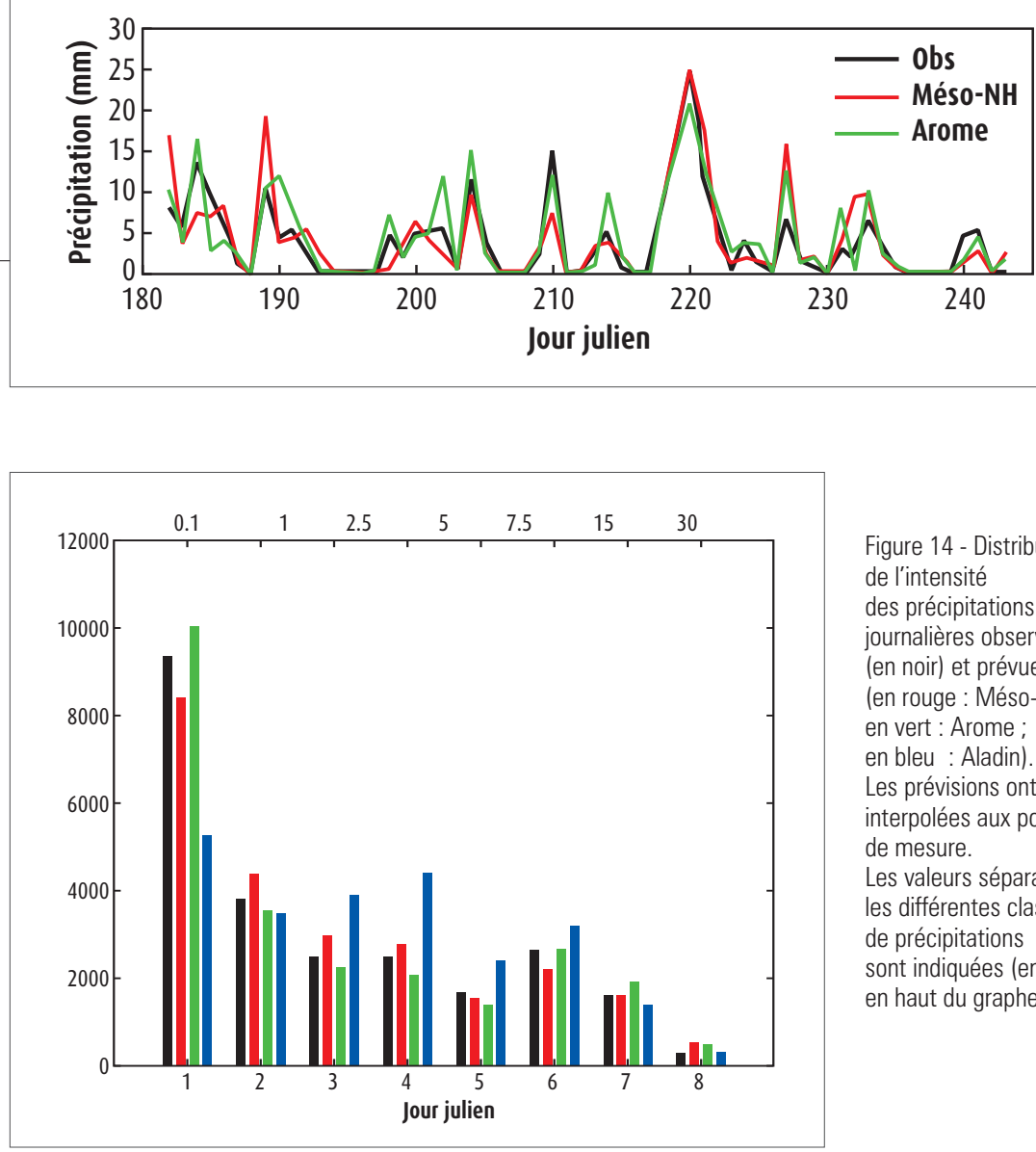

Figure 14 - Distribution de l'intensité des précipitations journalières observées (en noir) et prévues len rouge : Méso-NH : en vert : Arome ; en bleu : Aladin). Les prévisions ont été interpolées aux points de mesure.

Les valeurs séparant les différentes classes de précipitations sont indiquées (en mm) en haut du graphe.

temporelle des précipitations journalières, moyennées sur la région Cops, mesurées et prévues par les modèles A rome et $\mathrm{M}$ éso- $\mathrm{NH}$ au cours de juillet et août 2007. On y constate que les pics de précipitation sont bien reproduits par les deux modèles ; cela est vrai pour leurs occurrences, mais un peu moins sur leur intensité. II est particulièrement intéressant de constater que ces deux modèles, qui partagent les mêmes paramétrisations physiques, sont parfois en désaccord, ce qui met en évidence le très fort impact des conditions initiales et de couplage, dérivées dans un cas du système A rpège-A ladin et dans I'autre du système du CEPM M T.

Il est particulièrement intéressant de constater que les modèles à haute résolution sont bien plus à même de représenter la distribution des précipitations. A insi que I'indique la figure 14, le modèle opérationnel A ladin (de résolution spatiale $11 \mathrm{~km}$ et reposant sur

\section{Une contribution essentielle : I'assistance de prévisionnistes expérimentés}

Trois prévisionnistes (Julien Billault-Chaumartin, Jérôme Pauthe et Cédric Hertzog) de la Direction interrégionale Nord-Est de MétéoFrance se sont relayés pour assister les scientifiques au cours de la campagne. Leur expérience du terrain vosgien et leur savoir-faire ont été déterminants dans les prises de décision, particulièrement celles qui ont concerné les missions avion. À cette occasion, ils ont pu également confronter leur expérience et leurs outils (par exemple, la plateforme de visualisation Synergie en arrière-plan) à ceux de leurs collègues germaniques. 
I'approximation hydrostatique) sousestime les faibles précipitations et surestime les précipitations moyennes. $\mathrm{Ce}$ défaut est très significativement corrigé par les modèles non-hydrostatiques de haute résolution, tant pour A rome que pour M éso-N $\mathrm{H}$.

\section{Conclusions}

Bien qu'il soit un peu tôt pour tirer un bilan définitif des opérations conduites pendant la campagne, il semble acquis que la richesse et la qualité des observations recueillies au cours des mois de juillet et août 2007 permettra à la communauté Cops d'atteindre la majeure partie de ses objectifs. A insi que le sou- lignent volontiers nos collègues allemands, la base de données Cops est un véritable coffre au trésor. Gageons que nous saurons en exploiter les ressources au mieux, tant pour appréhender les mécanismes gouvernant les précipitations convectives que pour en mieux prévoir l'occurrence, l'intensité et la localisation.

\section{Remerciements}

$L$ a participation française au projet Cops a bénéficié du soutien financier de I'ANR, du Cnes, du programme L efe/ldao de I'Insu et de M étéoFrance. N ous remercions chaleureusement les personnels de l'Eost et de la DIRNE pour leur soutien logistique ainsi que I'équipe $4 M$ de $M$ étéoFrance et I'unité mixte CNRS/Cnes/ $M$ étéo-France Safire pour leur savoirfaire et leur professionnalisme en matière de campagne de terrain. Enfin, Cops n'aurait pas été possible sans I'engagement personnel de nombreux chercheurs, étudiants et techniciens auxquels nous tenons à exprimer nos remerciements et tout particulièrement à O. Caumont et V. Ducroca (CNRM / M icado), J. Escobar et D. Gazen (LA), D. B runeau, J. Pelon et $S$. B astin (SA), $P$. Tabary (M étéo-France), K. B oniface et P. Collard (GM), L. E stevan, D. Edouart et P.-H. Flamant (LM D), O. Bock et S. Nahmani (IGN), F. Blouzon et A. A bchiche (DT/Insu), D. Legain et O. Garrouste (CNRM /4M) et P. Gégout (IPGS).

\section{Bibliographie}

\footnotetext{
Bougeault P., P. Binder, A. Buzzi, R. Dirks, R. A. Houze, J. Kuettner, R. B. Smith, R. Steinacker et H. Volkert, 2001 : The MAP special observing period. Bull. Amer. Meteor. Soc., 82, 433-462.

Bouttier F., 2007 : Arome, avenir de la prévision régionale. La Météorologie, 8 série, 58, 12-20

Bruneau D., P. Quaglia, C. Flamant et J. Pelon, 2001 : The Airborne Lidar Leandre 2 for Water Vapor Profiling in the Troposphere. Part II: First Results. Appl. Opt., 40, 3462-3475.

Champollion C., F. Masson, M. N. Bouin, A. Walpersdorf, E. Doerflinger, O. Bock et J. Van Baelen, 2005 : GPS Water vapor tomography: Preliminary results from the Escompte field experiment. Atmos. Res., 74, 253274.

Champollion C., 0. Bock, C. Flamant, F. Masson et T. Weckwerth, 2009 : Mesoscale GPS tomography applied to the 12 June convective initiation event of IHOP 2002. Quart. J. Roy. Meteor. Soc., sous presse.

Cuesta J., E. Dimitri, M. Mimouni, P. H. Flamant, C. Loth, F. Gibert, F. Marnas, A. Bouklila, M. Kharef, B. Ouchène, M. Kadi et C. Flamant, 2008 : Multi- platform observations of the seasonal evolution of the Saharan atmospheric boundary layer in Tamanrasset, Algeria, in the framework the African Monsoon Multidisciplinary Analysis field campaign conducted in 2006. J. Geophys. Res., 113, D00C07, DOI:10.1029/2007JD009417.

Dabas A. et C. Flamant, 2002 : Les instruments de télédétection au sol. La Météorologie, 8 e série, 39, 76-82.

Doerflinger E., 2001 : Les applications météorologiques du système de positionnement satellitaire GPS. La Météorologie, 8érie, 34, 21-37.

Flamant C., F. Guichard, J. Van Baelen, O. Bock, F. Couvreux, D. Parsons, T. Weckwerth, J. Pelon, P. Drobinski, K. Lhomme, M. Guenno et E. Doerflinger, 2003 : La campagne IHOP 2002 : une campagne de mesure de la vapeur d'eau dans la couche limite. La Météorologie, 8e série, 43, 38-47.

Lafore J. P., J. Stein, N. Asencio, P. Bougeault, V. Ducrocq, J. Duron, C.Fisher, P. Héreil, P. Mascart, J. P. Pinty, J. L. Redelsperger, E. Richard et J. Vila - Guerau, 1998 : The MESO-NH atmospheric simulation system. Part I: Adiabatic formulation and control simulations. Annales Geophysicae, 16, 90-109.

Mauprivez M. et J.-P. Sandra, 2002 : Les profileurs de vent à vocation opérationnelle. La Météorologie, $8^{e}$ série, 39, 71-75.

Rotach M., M. Arpagaus, M. Dorninger, C. Hegg, A. Montani, R. Ranzi, F. Bouttier, A. Buzzi, G. Frustaci, K. Mylne, E. Richard, A. Rossa, C. Schär, M. Staudinger, H. Volkert, V. Wulfmeyer, P. Ambrosetti, F. Ament, C. Appenzeller, H.-S. Bauer, S. Davolio, M. Denhard, L. Fontannaz, J. Frick, F. Fundel, U. Germann, A. Hering, C. Keil, M. Liniger, C. Marsigli, Y. Seity, M. Stoll, A. Walser et M. Zappa, 2008 : MAP D-PHASE: Real-time Demonstration of - Weather Forecast Quality in the Alpine Region. Bull. Amer. Meteor. Soc., soumis.

Van Baelen J., Y. Pointin, F. Tridon, W. Wobrock et G. Peters, 2008 : PREPHIX : Precipitations and microphysical studies with a High resolution X-band radar: Calibration with a bin microphysical model and supporting measurements, Proceedings of the Fifth European Conference on Radar in Meteorology and Hydrology (ERAD2008), Helsinki, Finland, 30 juin - 4 juillet 2008.

Wulfmeyer V., A. Behrendt, H.-S. Bauer, C. Kottmeier, U. Corsmeier, A. Blyth, G. Craig, U. Schumann, M. Hagen, S. Crewell, P. Di Girolamo, C. Flamant, M. Miller, A. Montani, S. Mobbs, E. Richard, M. W. Rotach, M. Arpagaus, H. Russchenberg, P. Schlüssel, M. König,, V. Gärtner, R. Steinacker, M. Dorninger, D. D. Turner, T. Weckwerth, A. Hense et C. Simmer, 2008 : The Convective and Orographically-induced Precipitation Study: A Research and Development Project of the World Weather Research Program for improving quantitative precipitation forecasting in low-mountain regions. Bull. Amer. Meteor. Soc., DOI 10.1175/2008BAMS2367.1.
} 